\title{
El cambio de doxa en Chile \\ De la solidaridad al individualismo indiferente \\ Una lectura desde los discursos presidenciales pre y post dictadura
}

\section{Changing doxa: from solidarity to indifferent individualism. Reviewing presidential discourses in the pre and post dictatorial period in Chile}

Mónica Alejandra VARGAS AGUIRRE*

\begin{abstract}
Resumo: El artículo que se desarrolla a continuación devela por medio del análisis del discurso presidencial de 4 presidentes el cambio de doxa que se produjo en Chile después de la Unidad Popular y los efectos que esto tiene en el actual escenario político nacional.
\end{abstract}

Palavras-chave: Naturalización de la dominación. Política. Doxa. Dictadura. Imaginario social.

\begin{abstract}
This article analyses presidential discourses from four presidents in Chile. It aims at demonstrating the change of doxa, which emerged in the country after the 'Unidad Popular' and its effects on the current political arena.

Keywords: Establishment of the dominante. Politics. Doxa. Dictatorial period. Social imaginary.
\end{abstract}

* Académica, Universidad Academia de Humanismo Cristiano, Licenciada en Sociología y Licenciada en Trabajo Social, Magíster en Asentamientos Humanos y Medio Ambiente. mvargas@academia.cl 
La naturalización de la dominación es más peligrosa que una guerra abierta por más cruenta que esta sea. Cuando naturalizamos la dominación la guerra esta dentro de nosotros mismos y se manifiesta en nuestras contradicciones. (Mónica Alejandra Vargas Aguirre)

\section{Introducción}

Las relaciones de poder y de dominación no sólo tienen una manifestación estructural, está claro que existe también una dimensión simbólica que se ubica en el mismo nivel de importancia que la primera. El destacado sociólogo francés Pierre Bourdieu señala que "Todo poder admite una dimensión simbólica: debe obtener de los dominados una forma de adhesión que no descansa en la decisión deliberada de una conciencia ilustrada sino en la sumisión inmediata y prerreflexiva de los cuerpos socializados"1. Las relaciones que se producen en el espacio social chileno pueden ser analizadas entonces desde estas dos perspectivas, la estructural y la simbólica. Los análisis en el plano estructural son frecuentemente desarrollados en función de mejorar indicadores macro socioeconómicos, los análisis que hacen referencia al plano simbólico en cambio quedan generalmente en un segundo plano. El trabajo que se presenta a continuación pretende complementar la visión estructural de lo sucedido socioeconómica y políticamente en Chile antes y después de la dictadura de Pinochet, con un análisis del lenguaje utilizado en los discursos presidenciales del 21 de mayo de cuatro presidentes de la nación.

Se utilizará como eje teórico el concepto de doxa elaborado por Bourdieu, dado que éste permite entender de alguna manera la sumisión a la dominación simbólica. El autor desarrolla el concepto entendiendo por tal, un conjunto de creencias y de prácticas sociales que son consideradas normales en un contexto social, las cuales a su vez, son aceptadas sin cuestionamientos. Los orígenes sociales de estas creencias y prácticas, y sus principios de funcionamiento son desconocidos para los agentes, sin embargo, los criterios

1 Pierre, Bourdieu; “La dominación masculina," Editorial Anagrama, Barcelona, 1999. y formas de proceder promovidas por dichas creencias y prácticas son reconocidos habitualmente como socialmente válidos. Allí reside su eficacia simbólica: la doxa es una condición para mantener el estado de cosas existente en una sociedad sin que la sociedad misma lo cuestione.

La pregunta que rige este trabajo es ¿Cómo se manifiesta el cambio de doxa en el campo político, social y económico dentro del espacio social chileno pre y post dictadura?, la idea es iniciar el camino hacia una respuesta examinando los discursos presidenciales de cuatro personajes con la misma militancia en dos partidos políticos diferentes que se ubican en tiempos históricos distintos, dos antes de la dictadura y dos después de ella.

El objetivo es identificar los elementos diferenciadores de la doxa, por medio del análisis de conceptos representativos de los párrafos de los discursos del "21 de mayo" del primer año de gobierno de los dos presidentes pre dictadura y los dos presidentes post dictadura. Se analizaron por tanto los discursos presidenciales correspondientes a los mensajes del 21 de mayo del primer año de gobierno de Eduardo Frei Montalva, Democratacristiano y Salvador Allende Gossens, Socialista como representantes de la doxa pre dictadura y Eduardo Frei Ruiz-Tagle, Democratacristiano y Ricardo Lagos Escobar, socialista, como representantes de la doxa post dictadura. Se pretendió detectar conceptos que den cuenta de este cambio de doxa.

La hipótesis del presente trabajo es que ha habido un cambio dóxico en el espacio social chileno pre o post dictadura, en particular en el campo político. Este cambio va desde el reconocimiento de la solidaridad y lo colectivo definido como relevantes, a una concepción egoísta e individual de lo social puesta en primer plano, esto mediado por la imposición de un determinado modelo de sociedad que en principio se definió en el campo económico y que luego, con la autonomización de dicho campo se extendió al campo social, cultural y político. El ajuste estructural económico desarrollado durante la dictadura ha tenido su correlato en aquello aceptado como natural e incuestionable por la mayoría de los agentes que interactúan en los diferentes campos del espacio social del país.

Se trata de mostrar el cambio en la doxa dominante en el campo político de la sociedad 
El cambio de doxa en Chile De la solidaridad al individualismo indiferente...

chilena pre y post dictadura. Para esto se hará una comparación entre los discursos de los presidentes electos en los dos periodos inmediatamente anteriores al gobierno de Augusto Pinochet, es decir; Eduardo Frei Montalva, Demócrata Cristiano y Salvador Allende, Socialista, y compararlos con los dos presidentes electos en los dos periodos siguiente a la dictadura, es decir; Eduardo Frei Ruiz Tagle Demócrata Cristiano (como Frei Montalva e hijo de éste) y Ricardo Lagos Escobar Socialista (como Allende).

No se incluyó el gobierno de Patricio Aylwin en tanto se consideró un gobierno de transición demasiado permeado aun por los enclaves autoritarios, dado que la campaña y la votación se realizaron aun en tiempos del gobierno militar. Se consideró la comparación entre estos cuatro presidentes en tanto tienen la misma militancia política y el orden de sucesión en el poder es similar.

Se seleccionaron los discursos del 21 de mayo del primer año de mandato pensando que ellos reflejan la declaración de voluntades de un gobierno que recién se inicia y que cuentan con el respaldo político de un porcentaje importante de la población (dada la demostración de apoyo popular al ser electos), estos discursos por tanto permiten identificar aquellos planteamientos reconocidos por el imaginario electoral mayoritarios como válidos. Tradicionalmente es el discurso del 21 de mayo el que expone los lineamientos de la política anual de gobierno, es además, en este primer discurso, donde se explicitan los lineamientos para el periodo completo y esto a su vez refleja la propuesta de la candidatura y por tanto es reflejo de la doxa dominante en el espacio social.

\section{La doxa pre y post dictadura}

Para Bourdieu la sociedad está constituida por un espacio social objetivo que él denomina sistema de posiciones y campos, sumado a un espacio de representaciones simbólicas que contienen disposiciones cognitivas y comportamentales incorporadas, que el autor define como habitus de los agentes. En este contexto la práctica se constituye entonces en una toma de posición de los agentes que implican disposiciones específicas.
Doxa es un término griego que se puede traducir como "gloria" o "fama" aunque también como "opinión", es esta última acepción la que hace referencia a aquel conocimiento que no necesariamente es cierto, pero que es la percepción masificada circulante de la realidad. "En este sentido parecen utilizarlo tanto Parménides, al distinguir la "vía de la verdad" de la "vía de la opinión", como Platón, al distinguir, también contraponiéndolas, la "doxa" de la "episteme", es decir, el conocimiento aparente, (el conocimiento de la realidad sensible), del verdadero conocimiento, (el conocimiento de la verdadera realidad, de las Ideas)" 2 .

Bourdieu define doxa como aquellos esquemas cotidianos, no reflexionados y considerados como naturales, aquello admitido per se, en tanto es parte de lo habitual y que no requiere de una reflexión, es lo no cuestionado, el habitus colectivo que predomina en un determinado periodo de tiempo en una sociedad específica. La doxa es el sustento irreflexivo de las acciones de los actores en sociedad. La doxa puede cambiar rápidamente en sociedades permeables al influjo externo, o mantenerse inalterable en sociedades conservadoras, si, cambia de un periodo a otro y la velocidad de sus cambios depende del tipo de sociedad de que se trate, pero también depende de los hitos, acontecimientos históricos que marquen esa sociedad y que pueden generar alteraciones doxicas. En Chile, por ejemplo, el golpe de estado de 1973 y el largo periodo represivo que le siguió, produjo alteraciones de tal magnitud en las prácticas cotidianas de los chilenos que poco a poco se fueron internalizando, transformándose lentamente en una modificación de la doxa anterior. Dicha transformación implicó que se hiciese innecesaria una dominación explícita realizada por la fuerza ya que aquellos preceptos impuestos por la dictadura pasaron a ser parte de la cotidianidad del país, ya nadie cuestiona que el modo de vida chileno es aquel regido por el neoliberalismo, ya nadie se pregunta qué nos pasó como sociedad y por qué nos hemos vuelto más egoístas, más violentos y menos solidarios.

2 Diccionario de filosofía http://www.filosofia.com.mx/index.php?/ perse/archivos/la_jerga_del_filosofo4/ Página con licencia creativecommons.org/. 
La doxa funciona de modo sutil "a través del lenguaje, a través del cuerpo, a través de las actitudes hacia las cosas, que están por debajo del nivel de la conciencia"3 esto la hace tan difícil de resistir y por tanto de modificar. La doxa es teoría a nivel del inconsciente que orienta las prácticas y las interpretaciones del mundo.

\section{Las diferencias en la doxa económica, social y política pre y pos dictadura.}

\section{Cambio de Doxa en el Campo Económico}

\section{La organización de la producción}

El campo económico en la etapa pre dictadura era considerado parte del espacio social pero su preponderancia era menor respecto al campo político. El campo económico durante la dictadura impone sus conceptos de eficacia, leyes de mercado, competencia, maximización de la inversión etc., poco a poco se va autonomizando, adquiriendo una importancia particular por sobre los otros campos, para consolidarse en la etapa post dictadura como un elemento central de la doxa dominante en el espacio social chileno. El capital económico más que el capital cultural o político marca las relaciones en el espacio social chileno.

La economía en el periodo pre dictadura estaba en función del bienestar de la población. Un cambio no menor en la institucionalidad económica fue el que se plasmó en la reforma a la constitución de 1925, el 20 de enero de 1967, fecha en la cual se estableció la existencia de "un nuevo concepto de la función de la propiedad. La función social de la propiedad comprende cuanto exijan los intereses generales del Estado, la utilidad y salubridad, el mejor aprovechamiento de las fuentes de energías productivas en el servicio de la colectividad, y la elevación de las condiciones de vida del común de los habitantes"4. Este cambio en la concepción de la propiedad privada venía a plasmar en el ordenamiento institucional del país un sentimiento de la sociedad completa. Nótese la idea de que la propiedad está mediada por las necesidades de la población en su conjunto y regulada por el Estado, esto era entonces otro de los elementos que permitían configurar la doxa de la época.

En el discurso pre dictadura el presidente Eduardo Frei Montalva se refiere a lo público como lo chilenizado, es la organización pública, lo perteneciente al pueblo. Allende por su parte utiliza la palabra "popular" para referirse a lo público, la que se repite 23 veces en su primer discurso del 21 de mayo de 1970, de hecho plantea que su gobierno "tiene su origen en la voluntad popular libremente manifestada. Sólo ante ella responde, los movimientos y partidos que lo integran son orientadores de la conciencia revolucionaria de las masas y expresión de sus aspiraciones e intereses. Y también son directamente responsables ante el pueblo5", esto refleja la preponderancia de la organización de la civilidad por sobre lo privado.

En el periodo post dictadura en cambio, Eduardo Frei Ruiz-Tagle se caracterizó por darle una gran relevancia al sector empresarial en el país, de hecho él mismo era un empresario que asumía el gobierno, no es de extrañar entonces que el concepto "Privado" se repita 27 veces en el discurso presidencial analizado. Frei Ruiz Tagle planteaba que había abandonado sus empresas para dedicarse a la política, sin embargo, mantuvo importantes capitales y activos en Inversiones Saturno S.A., en la cual participaba Alberto Coddou, quien a su vez era parte del directorio de PROVIDA que a su vez es accionista de ENDESA, que es dueña de la Central Ralco, que él aprobó durante su mandato, en contra incluso del director de la CONADI y no pocos funcionarios de la CONAMA que habían rechazado el estudio de impacto ambiental un año antes de que el presidente autorizara su construcción, que dicho sea de paso fue autorizada por la contraloría el último día en que Frei fue presidente, liderando a la coalición que luego volverá a ganar las elecciones. La costanera norte es otro ejemplo flagrante de trasgresión del presidente en beneficio de los empresarios. Un proyecto rechazado por la CONAMA luego aparece aprobado.

\footnotetext{
3 Bourdieu, Pierre \& Eagleton, Terri "Doxa y vida ordinaria".

4 Ibíd.. Página 216.
} 
El cambio de doxa en Chile De la solidaridad al individualismo indiferente...

El vínculo con el empresariado, las constantes transgresiones al medio ambiente, la preponderancia de los empresarios en desmedro del pueblo (contrario a lo que había realizado su padre con su "revolución en libertad") todos los indicadores objetivos apuntaban a que gobernaba para la elite económica, sin embargo, no era cuestionado mayoritariamente, sólo algunas voces desde la izquierda extraparlamentaria (como si existiera otra izquierda) se atrevían a plantear el problema. También desde los medioambientalistas y las organizaciones indígenas hubo alertas, pero las personas en su conjunto ("el pueblo" o "la gente" dependiendo del discurso) no reaccionaba, para el imaginario social era correcto o cuando menos no cuestionable que un empresario hiciera negocios mientras conducía el país. Triunfo rotundo para la doxa neoliberal continuidad de la dictadura, ya no es necesario ocultarse, la dominación simbólica en su más clara expresión. El concepto privado prima en el primer año de gobierno de Frei.

Un párrafo representativo de su discurso es "No habrá crecimiento económico sin una cuota de esfuerzo y sacrificio. Un mayor crecimiento sólo será posible en tanto seamos capaces de lograr sustanciales incrementos en el ahorro y la inversión del país. Esto nos obliga a realizar un esfuerzo de austeridad -tanto público como privado, postergando gastos corrientes muchas veces justificables, para destinarlos al ahorro y así financiar la inversión"6.

A pesar de que el mandato de Frei había privilegiado a los grandes empresarios, es electo Ricardo Lagos Escobar de la misma coalición, cuestión que hubiese sido impensable antes de la dictadura. El pueblo hubiese castigado en las urnas al presidente empresario que dejó sin tierras a los indígenas del sur.

Lagos por su parte, perteneciente a las filas del partido socialista, es el fundador del PPD, Partido Por la Democracia, que es algo así como socialistas y otros. Pero este presidente otrora economista doctrinariamente socialista menciona 5 veces lo privado y 16 veces lo público. Nótese el cambio de énfasis discursivo respecto a Frei Ruiz

\footnotetext{
6 Frei Ruiz-Tagle, Eduardo; "Mensaje de presidencial, 21 de Mayo del año 1994", Gobierno de Chile Página 8.
}

Tagle, este puede deberse a las raíz socialista de Lagos y las características de plasticidad de su personalidad que dan origen a "sujetos sucesivos presentes en su evolución ideológica"7. Pero, en lo sustancial la diferencia no existe. Esto se ve evidenciado en el enfoque de su gobierno dada la preponderancia que le da a las empresas en la organización de la producción en desmedro de las personas y del medio ambiente, prueba de esto último fueron las declaraciones de las organizaciones medioambientales en contra de su nominación como enviado especial para el cambio climático de Naciones Unidas.

En cuanto a la manera de organizar la producción, las citas evidencian claras diferencias en la utilización de los conceptos para referirse a ella la producción, en el gobierno de Allende se habla del "Socialismo nuevo latinoamericano", así habla Allende cuando se refiere a ello: "nuestra tarea es definir y poner en práctica como la vía chilena al socialismo, un modelo nuevo de Estado, de economía y de sociedad, centrado en el hombre, sus necesidades y sus aspiraciones"8

Es notoria la ruptura con la doxa anterior en el caso de Frei Ruiz-Tagle ya que en este punto sólo hace referencia a lo empresarial y no aparece haciendo referencias claras a lo colectivo, a no ser, como veíamos anteriormente, para hacer alusión al necesario esfuerzo colectivo por mejor las inversiones y el crecimiento del empresariado en el país. De hecho la única vez que utiliza el concepto colectivo lo hace en razón de los "convenios colectivos" en las ISAPRES que en el país se reconocen como un gran negocio.

Lagos por otra parte alude a las empresas y el empresariado 16 veces, pero tampoco habla de lo colectivo, es más, Lagos no pronuncia en todo su discurso la palabra socialismo. Otra conquista de la doxa post dictadura, un presidente socialista elegido después del golpe de estado de 1973 que derrocó a un correligionario suyo, no pronuncia ni una sola vez la palabra vedada por casi todo el periodo de la dictadura, "socialismo".

\footnotetext{
7 Mella Marcelo; "Carácter y transformación del liderazgo de Lagos" Instituto Internacional de Gobernabilidad de Cataluña, colección de documentos, Cataluña, España página 3.

8 Allende Gossens, Salvador; "Mensaje Presidencial al Congreso Pleno, 21 de mayo de 1970", Página 6.
} 


\section{Recursos naturales cobre y forestales}

La nueva doxa tiene como elemento central la búsqueda de beneficio a corto plazo y esto es lo que rige todas las elecciones económicas ${ }^{9}$, no existe por lo tanto proyección ante las posibles catástrofes derivadas de la explotación irracional de los recursos naturales, dentro de estos el cobre (recurso no renovable) y los recursos forestales.

En la época de Frei Montalva el cuidado del medio ambiente no era prioritario, lo prioritario era el desarrollo económico del país, Frei se refiere por ejemplo a la explotación de los recursos naturales de la siguiente manera "mi gobierno ha considerado que la explotación de sus recursos mineros para fines de exportación es la principal palanca que puede aplicar para obtener en forma rápida un cambio en las condiciones de nuestro comercio exterior y de nuestra balanza de pagos. En consecuencia su política no es la de reservar esos recursos para el futuro sino la de aplicarlos hoy en la más grande medida para la expansión de nuestra economía y el bienestar de nuestro pueblo"10, sin embargo en otra parte del discurso hace referencia a la deforestación y a la erosión aunque su preocupación sigue siendo económica cuando señala que "estamos poniendo en ejecución un plan de reforestación que permita además controlar la erosión, regular las fuentes de agua y dar un poderoso impulso a las industrias que utilizan la madera como materia prima"11.

Frei Montalva creía que la inversión extranjera es una opción ante la carencia de recursos para explotar el cobre que existía en el país, sin embargo, hacía la salvedad, consecuente con la doxa pre dictadura, que se debía asegurar el consumo interno y el control de la producción y de sus beneficios por parte del Estado, esto se traduce en la chilenización y nacionalización pactada que significó en la práctica que el Estado tuviera un control del $51 \%$ de las acciones del cobre, anteriormente controlada por capitales

\footnotetext{
9 Bourdieu, Pierre; "El sociólogo y las transformaciones recientes de la economía en la sociedad", editorial Libros de Rojas, Universidad de Buenos Aires, 2000. página 23.

10 Eduardo Frei Montalva, Discurso presidencial 21 de mayo de 1965.

11 Frei Montalva Página 58.
}

norteamericanos y la creación de CODELCO.

Allende por su parte hace referencia a la explotación de los recursos naturales sólo una vez, recordando que estos son de todos los chilenos planteando: "Las normas institucionales en que se basa la explotación extranjera de los recursos naturales de Chile fueron aquí establecidas. Pero este mismo Parlamento las revisa, ahora, para devolver a los chilenos lo que por derecho les pertenece"12, este párrafo deja entrever la relevancia que tenía en términos de propiedad más que ecológico el tema.

En el contexto pre dictadura el cobre es un icono de soberanía, Allende al igual que su antecesor advierte la necesidad de asegurar recursos energéticos que permitieran hacer avanzar a la nación y así se refiere al cobre; "A lo avanzado en la liberación de las energías chilenas para reedificar la nación, tendrán que seguir pasos más decisivos. A la Reforma Agraria en marcha, a la nacionalización del cobre que sólo espera la aprobación del Congreso Pleno, cumple agregar, ahora, nuevas reformas"13. Este presidente da por asegurada la nacionalización del cobre, y así sucede, el congreso aprueba tiempo después la ley de nacionalización del cobre. La doxa pre dictadura no concebía este recurso mineral en manos extranjeras aunque si su explotación irracional en función del crecimiento económico. Queda evidenciado aquí que dentro de los conceptos naturalizados antes de la dictadura la idea de la ecología no aparecía como relevante, en muy pocos discursos aparece la protección del medio ambiente como fundamental a pesar de que ya en 1970 existía el informe de Roma alertando acerca del impacto del crecimiento económico a costa de la explotación de los recursos naturales en el medio ambiente.

En el periodo post dictadura, cuando Frei Ruiz Tagle se refiere al cobre señala: "El principal compromiso que hoy tenemos respecto a CODELCO, es volver a posicionar la empresa entre los productores de cobre de más bajos costos del mundo. El país necesita que sea un muy buen

\footnotetext{
12 Salvador Allende Goznes, Discurso presidencia 21 de mayo de 19.

13 Salvador Allende Gossens.
} 
El cambio de doxa en Chile De la solidaridad al individualismo indiferente...

negocio para Chile y que esté al servicio de todos los chilenos"14. De aquí se puede leer que para este presidente el cobre debe ser explotado de manera extensiva en tanto eso permita su producción a bajo costo.

Por otra parte habla de la modernización del sector, lo que se tradujo en una apertura aun mayor a los privados y a las trasnacionales a la explotación de este recurso. Así señala que la única forma de explotar el recurso es con capitales extranjeros y privados, y dice "En los últimos cuatro años se logró detener la tendencia al alza de los costos, reducir dotaciones, poner en marcha un importante esfuerzo de inversión en exploración geológica e investigación y desarrollo, y abrir canales para la exploración y explotación conjunta con el sector privado de los yacimientos que no son necesarios para garantizar la viabilidad futura de las actuales divisiones mineras". Atrás quedó la vieja doxa que propiciaba la chilenización del cobre porque este era un recurso de todos los chilenos y sus beneficios debían llegar a toda la población. En este periodo, sin embargo, la sociedad en general tiene un mayor conocimiento de los problemas medio ambientales que la producción de cobre provoca (contaminación de napas subterráneas, destrucción de nichos ecológicos, etc.), sin reaccionar.

En cuanto al recurso forestal, este también es mencionado en función de la economía y no del equilibrio ecológico, Frei plantea "Hemos iniciado en estos días el Programa de Diversificación de la Silvicultura Nacional, que ampliará la oferta de especies forestales existentes, a la vez que empezará el cultivo de nuevas especies que ofrecen amplias perspectivas de crecimiento15".

Como se veía anteriormente, Frei Ruiz Tagle no tuvo una preocupación por el medio ambiente, de hecho inundó varias hectáreas de terreno en el sur y pasó encima de varios informes negativos de la CONAMA, esto a pesar de que fue durante su mandato que se dicta el Reglamento de Evaluación de Impacto Ambiental de la Ley de Base del Medio Ambiente.

\footnotetext{
14 Frei Ruiz-Tagle, Eduardo; "Mensaje de presidencial, 21 de Mayo del año 1994", Gobierno de Chile Página 46.

15 Frei Ruiz-Tagle, Eduardo; "Mensaje de presidencial, 21 de Mayo del año 1994", Gobierno de Chile Página 39.
}

En el caso de Lagos, este no hace grandes referencias en su discurso al tema, su gobierno, al igual que el de Frei Ruiz Tagle se destaca por la construcción de grandes obras viales e hidroeléctricas.

Sin embargo un grave problema ecológico se produjo en el sur con la instalación de una industria de celulosa, la empresa Celulosa Arauco fue acusada de ser la causante de la muerte de cientos de cisnes de un santuario de la naturaleza ubicado en Valdivia. Celulosa Arauco produce riles considerados tóxicos, sin embargo hoy en día se encuentra en pleno funcionamiento y apenas ayer un inexplicable incendio consumió años de informes de investigaciones respecto al impacto de la celulosa. La noticia pasa casi desapercibida entre la farándula y el sensacionalismo mediático creado en torno a la delincuencia común pasando desapercibida la delincuencia de alto impacto nacional como la de las plantas de celulosas que a la larga genera más muerte y destrucción.

El tema medioambiental no era parte del imaginario social pre dictadura, lo social era preponderante respecto a lo medioambiental, sin embargo, un elemento central de la doxa anterior era el respeto por el ser humano, a pesar de la violencia política, a pesar de las grandes crisis que vivió el país, el respeto por el ser humano era lo fundamental, cuestión que se rompe una vez más con la dictadura donde el ser humano pasa a se un recurso más en la producción y donde su valor como persona es medido por indicadores macro económicos.

En suma ni antes ni después de la dictadura la población del país ha tenido internalizada la idea de protección del medio ambiente como elemento fundamental para el mantenimiento de la humanidad.

\section{Concepto utilizado para referirse a la evolu- ción económica}

Frei Montalva utiliza fundamentalmente el concepto desarrollo para referirse a la evolución económica del país. En su discurso a la patria joven del 21 de junio de 1964 señala "Con ustedes vamos 
a construir el desarrollo económico de Chile. Vamos a levantar la condición de la agricultura chilena, para que la tierra alimente al pueblo de Chile. Esta será una tarea de la más alta prioridad en mi gobierno. Vamos a desarrollar la industria. Chile tiene un definido destino industrial por la calidad de sus trabajadores, sus materias primas y por su tradición de nación laboriosa."16. Si bien esta idea de desarrollo económico se centra en lo social, no es menos cierto que es pensado en función del desarrollo social del pueblo chileno (si escribí pueblo) y la prueba está en las nacionalizaciones y la reforma agraria. Para ser presidente debía existir un compromiso con el crecimiento económico del país pero ligado a valores como la solidaridad y el respeto por los derechos humanos.

En el caso de Allende, este utiliza el concepto generalmente para referirse al desarrollo social del país más que a la economía, de hecho sólo utiliza una sola vez el concepto para referirse al ámbito productivo aunque claro está este también era un espacio de preocupación.

Frei Ruiz-Tagle en cambio utiliza indistintamente crecimiento y desarrollo, la connotación que tienen en su discurso va desde lo puramente económico al lo social haciendo difícil descifrar su concepción de desarrollo.

Lagos Escobar rescata el concepto de desarrollo dentro de la nueva doxa identificando las deficiencias que tiene el mero crecimiento económico se propone revertir la situación y en su primer discurso declara creer "en el libre comercio"17 aunque continua señalando que también conoce los subsidios de los países desarrollados.

En otra parte de su discurso también refuerza la idea de que el crecimiento económico es indispensable para el bienestar, al referirse al desarrollo tecnológico del país señala "Nadie debe quedar sin acceso al bienestar que surja del crecimiento económico y de la incorporación de Chile a la revolución tecnológica ${ }^{18}$. Ejemplos que vinculan desarrollo a crecimiento económico en el discurso de Lagos se pueden encontrar muchos,

\footnotetext{
16 Frei Montalvo, Eduardo; "Discurso a la Patria Joven", 21 de junio de 1964.

17 Lagos Escobar Ricardo; página 22

18 Lagos Escobar Ricardo, Página 6
}

esto da cuenta de que la doxa dominante en chile durante su gobierno naturalizaba la idea de que no es posible el desarrollo social sin crecimiento.

\section{Cambio de Doxa en el Campo Político}

Según Bourdieu en el campo político el interés en juego es el poder político y los dominantes intentan mantener una lógica de equilibrio político que les es favorable para el ejercicio de su dominación. En los casos en los que estamos comparando se evidencia un cambio en el equilibrio de fuerzas en el campo político, en la etapa previa a la dictadura este campo era dominado por la participación entusiasta y espontánea de la población en la construcción de su propio futuro, los partidos políticos, movimientos sociales y organizaciones comunitarias cumplían un rol central en este campo. La dictadura rompió esa lógica dentro del campo político chileno, encarceló a sus líderes e hizo un trabajo subliminal de desprestigio de la política, el gobierno militar, dueño de toda la fuerza real y simbólica generó en la población una profunda desconfianza de aquellos que participan activamente en este campo. A partir de ahí los problemas de la polis se resuelven en el campo económico y aquellos que quieren participar en lo político primero deben ser ciudadanos en lo económico, se comienza a desarrollar ahí el concepto de Estado subsidiario que viene a reemplazar al Estado de Bienestar naturalizado en la doxa pre dictadura.

Un estado subsidiario tiene como objetivo central la incorporación de los individuos al mercado, el subsidio consiste en un primer paso para que luego cada uno camine solo por el mercado. Se define como aquel en que "La asignación de recursos en el marco de la política social está dada por la disminución del gasto fiscal por medio de la privatización de los servicios sociales de los estratos medios altos y de una focalización de los escasos recursos en la población más pobre la que debe esforzarse por hacer un aporte en la satisfacción de sus necesidades, es decir, el financiamiento es la mayoría de las veces compartido, asignándole al sector privado el rol de proveedor de bienes 
El cambio de doxa en Chile De la solidaridad al individualismo indiferente...

y servicios transables en el mercado, lo que se traduce en un subsidio a la demanda"19.

Otro cambio relevante hace referencia a aquello que es señalado por Lechner como el "desplazamiento de los límites de la política", mientras antaño el poder político se caracterizaba precisamente por crear su propio espacio, la nación, el actual proceso de globalización no sólo permea las fronteras nacionales como nunca antes, sino que socava la relativa congruencia que existía entre los espacios políticos, económicos y culturales. Por consiguiente, tanto la soberanía nacional como la categoría de soberanía popular , tan crucial como la idea democrática, se ha vuelto problemática ${ }^{20}$ y se ha vuelto problemática porque se han naturalizado irreflexivamente ideas y patrones de conducta que no son propios de la sociedades latinoamericanas.

Como se señaló anteriormente la doxa pre dictadura concebía al Estado como un Estado Benefactor en tanto "orienta las políticas sociales en función de una mayor equidad y justicia social con una visión redistributiva generalizada a toda la población y con alto porcentaje comparativo del PIB asignado al gasto social.

La postura frente al abordaje de los problemas que impiden el desarrollo está orientada a permitir una cobertura universal por medio de la asignación de una gran cantidad de recursos a la implementación de la política, lo que se traduce, al ponerlo en términos de mercado, en un subsidio de la oferta"21. Por el contrario, la idea de Estado instalada en la Doxa post dictadura es un estado subsidiario en el cual las orientaciones políticas están en función de los equilibrios macroeconómicos y del mantenimiento de un gasto social moderado.

\footnotetext{
19 Vargas Aguirre Mónica; "Políticas Sociales y Trabajo Social un análisis histórico desafíos, dilemas y propuestas", Ponencia presentada al Congreso Internacional "Políticas Sociales para un Nuevo Siglo", Universidad del Bío Bío, 21 al 24 de Noviembre, Concepción Chile. Publicada en: http://www.ubiobio.cl/cps/ponencia/doc/ p14.5.htm

20 Lechner Norbert; "Los nuevos perfiles de la Política", revista nueva sociedad №30, 1994. páginas 180-181, página 270.

21 Ibíd.. Página 2
}

\section{Rol asignado al Estado}

Para Frei Montalva el rol del Estado es el de un estado benefactor que debe satisfacer las necesidades de su pueblo, pero también concibe al Estado no aparte del pueblo sino a este como elemento fundamental en el accionar público. Sin embargo, plantea la relevancia del pluripartidismo y señala "somos enemigos del sistema de partido único, y como demócrata creemos en las elecciones libres y periódicas y en la posibilidad y el derecho de las minorías, si es esa la voluntad del pueblo para convertirse mañana en mayorías", de aquí se desprende que una parte de la población del país está preocupada por el avance de una ideología contraria a la norteamericana en la cual el partido único es una alternativa tan válida y democrática como el pluripartidismo. De ahí sale por cierto la votación de Frei Montalva, el cual de no existir esta amenaza probablemente no hubiese salido presidente.

Allende Gossi en cambio señala que la "tarea es definir y poner en práctica como la vía chilena al socialismo, un modelo nuevo de Estado, de economía y de sociedad, centrado en el hombre, sus necesidades y sus aspiraciones"22. El presidente concibe el Estado al servicio del pueblo y un cambio radical respecto a la idea anterior de gobierno. El intento de dar mayor representación al pueblo y disminuir la influencia de las élites en la conducción del Estado

Lagos Escobar a pesar de su historia continúa con la lógica de la dictadura y defiende un Estado subsidiario, en su discurso señala en varias ocasiones la necesidad de ajustar los gastos o de "asignación más eficiente de los fondos públicos" 23 para mejorar los programas sociales, teniendo siempre claro que en su discurso propone "hacer de la responsabilidad fiscal un elemento central"24 durante sus 6 años de gobierno.

Lagos permanentemente privilegia al mundo empresarial en desmedro del mundo social, como muestra de ello durante su periodo "El Poder Legislativo fue mucho más eficiente al tramitar

\footnotetext{
22 Allende Página 6

23 Lagos Escobar: página 11

24 Lagos Escobar: página 11
} 
leyes que benefician al mercado y a sus dueños, que al mundo social: la reforma al mercado de capitales -centro de la Agenda Pro Crecimiento demoró cuatro meses para su aprobación; el TLC con Estados Unidos, dos meses; la Ley Corta de Pesca, un mes; y la eliminación de la doble tributación entre Chile y España, un día. La nueva Ley del Consumidor tardó 32 meses y el Chile Solidario, 18 meses $^{25}$. La idea es potenciar el desarrollo del capital para así incorporar una mayor cantidad de personas al mercado, el presidente señala "los invito a expandir al máximo nuestra capacidad económica, para que esa parte de la familia chilena que sufre la pobreza se siente también a la gran mesa común a compartir los frutos de la nación"26.

\section{Relaciones Internacionales favorecidas}

El vínculo establecido internacionalmente por los presidentes en los periodos comparados muestra que en los casos de Allende se hace alusión a Latinoamérica como continente privilegiado en los vínculos en general en su discurso. Esto es correspondiente a la doxa dominante en tanto durante su periodo en donde las relaciones con el resto de los países del continente resulta relevante en el imaginario social.

Frei Montalva a pesar de haber reanudado las relaciones diplomáticas con la Unión Soviética (después de que habían sido cortadas por Gabriel González Videla en los albores de la guerra fría), privilegia en la práctica el vínculo con Estados Unidos en el contexto de la Alianza Para el Progreso que John Kennedy propuso para frenar la influencia de la revolución Cubana en América Latina ya en plena guerra fría, en este sentido señala: "de Estados Unidos hemos recibido una colaboración amplia y decidida y una cabal comprensión de nuestro programa de desarrollo económico y de transformaciones sociales. En el gobierno y en los sectores privados de esta nación"... y continua "los órganos directivos de la Alianza para el Progreso han colaborado desde el

25 Diario La Nación, 26 de Septiembre de 2004".

26 Lagos Escobar Ricardo; Discurso Presidencial del 21 de mayo de 2000. página 2. primer momento con mi gobierno en una forma que compromete también nuestro reconocimiento"27 . En su discurso también, al igual que Allende señala que las relaciones relevantes son aquellas que se establecen con el resto de los países de América Latina y dice "Consideramos como objetivo fundamentadle la política exterior de Chile la integración latinoamericana" ${ }^{28}$, esta frase también es un parte de la doxa de la época, se hacía impensable un mensaje presidencial que no hiciese alusión a la unidad latinoamericana. En otra parte del mensaje Frei Montalva se refiere también a Europa, sus relaciones con el viejo continente son por todos conocidas.

En el caso de Frei Ruiz-Tagle y Lagos Escobar la mención a las relaciones internacionales es mucho más frecuente. En el caso de otros continentes en Allende no aparecen mencionados, Frei Montalva reconoce a Estados Unidos, Europa y Asia, Frei Ruiz-Tagle menciona a Europa, Estado Unidos y Asia, apareciendo este último continente como parte constituyente de la nueva doxa planetaria que reconoce la importancia de las relaciones internacionales como un elemento central en la política de un país.

La mención que hace Allende del continente latinoamericano tiene relación con la construcción conjunta de un nuevo ideario continental, así plantea; "Pero aquí y ahora, en Chile y en América Latina, tenemos la posibilidad y el deber de desencadenar las energías creadoras, particularmente de la juventud, para misiones que nos conmuevan más que cualquier otra empresa del pasado". Frei Montalva en cambio señala al referirse a la importancia de la OEA "No nos negamos a considerar el hecho de que hay quienes, despreciando la democracia, pretenden utilizar la subversión contra la ley y el orden jurídico como el instrumento para apoderarse del poder", en abierta alusión a Cuba y al avance de los proceso revolucionarios del continente.

Al margen de que Frei y Allende no tuviesen posturas similares respecto a la unidad latinoamericana, ambos la plantean en su discurso

\footnotetext{
27 Frei Montalva Página 96.

28 Frei Montalva, Eduardo; "Primer mensaje del presidente de la república de chile", 21 de mayote 1965 Página 94.
} 
El cambio de doxa en Chile De la solidaridad al individualismo indiferente...

recogiendo por cierto el sentir ciudadano de la época.

Para Frei Ruiz -Tagle lo fundamental en las relaciones internacionales son los lazos económicos, aunque también entiende necesarias las relaciones de paz entre los países, pero en cada párrafo resalta lo económico, como muestra este párrafo que refleja la esencia de su discurso en lo internacional "Los vínculos con América Latina ocuparán un lugar prioritario en nuestra política exterior. América Latina ha vuelto a convertirse en una de las áreas de mayor dinamismo económico en el mundo. Se hacen, entonces, más necesarios que nunca la complementación y la integración económicas".

La referencia de Lagos al tema constata por una parte al nuevo orden mundial y dentro de éste a las relaciones económicas, más que a un concepto de solidaridad continental o a la construcción de una nueva sociedad que de cuenta de las particularidades del continente, Lagos habla así "hemos dado prioridad a nuestras relaciones con América Latina y en especial a los países del MERCOSUR. Porque me parece esencial la política exterior, una vez que los países nacen a partir de su realidad regional. En este mundo que se está articulando si no hablamos con una sola voz, no seremos oídos. Para hablar en este mundo y resolver donde se discuten las nuevas normas, quién las discute, cómo las discute, de qué carácter son en el orden económico y regulatorio internacional, en esta aldea global, quién va a fijar las normas, cómo nos incorporamos en ese debate, como el pequeño país que somos"

\section{Mecanismos de integración}

En ninguno de los textos se encontró una referencia explicita al modo específico de integración al ámbito internacional, sin embargo, se puede extraer de ellos la esencia y hacer una clasificación.

La Doxa predictadura enfatiza el vínculo social y político latinoamericanista no sólo en Chile sino que también en el resto del continente, esto implica la existencia de un clima de hermandad latinoamericana que, a pesar de los siempre presentes conflictos fronterizos, en el imaginario social hace sonar particularmente el sentido que Bolívar instauró.

En el caso de Allende el contenido nos habla de una integración política con un fuerte acento en la idea de un continente unificado con un proyecto común cuya esencia es la integración de los excluidos, así en su discurso plantea; "el pueblo necesita abrigar sus familias en casas decentes, con un mínimo de facilidades higiénicas, educar a sus hijos en escuelas que no hayan sido hechas sólo para pobres, comer lo suficiente en cada día del año, el pueblo necesita trabajo, amparo en la enfermedad y en la vejez, respeto a su personalidad. Eso es lo que aspiramos a dar en un plazo previsible a todos los chilenos. Lo que ha sido negado a América Latina a lo largo de siglos. Lo que algunas naciones empiezan a garantizar ahora a toda una población"29.

Frei Montalva también se hace eco de este sentido de integración social y política sumando la relevancia de la integración económica, así señala "Debemos hacer un esfuerzo creativo para darle contenido y forma a América Latina como expresión de un mundo nuevo donde el progreso se realice en libertad, la convivencia se practique bajo la ley y la justicia tenga una expresión plena" 30 y luego sigue señalando la importancia de tratados comerciales a nivel continental, cuando habla de un estudio encargado a Prebish, Mayobre Sanz y Santa María, cuya conclusión fue la creación de un mercado común latinoamericano. Las creencias y prácticas sociales de la época no permitirían otra cosa en un discurso presidencial, era deber por tanto dar cuenta de ellas, el mercado latinoamericano resultaba central, aunque en Frei Montalva aparece incipientemente el fomento de las relaciones económicas con el resto del mundo.

La doxa post dictadura evidencia un quiebre en el sentido de las relaciones con América Latina puestas en el centro con anterioridad, la racionalidad con arreglo a fines se instala también en el plano de las relaciones internacionales desplazando la racionalidad con arreglo a valores, Frei Ruiz -Tagle representa fielmente este cambio dóxico.

\footnotetext{
29 Allende Grossi página 6.

30 Frei Montalva página 94
} 
Como se veía anteriormente, este presidente entiende el vínculo internacional con un énfasis en lo económico, en uno de los párrafos de su discurso plantea; "mantendremos hacia nuestros vecinos una política de acercamiento constructivo e integración física y económica." ${ }^{11}$, en su discurso también señala la necesidad de asegurar las buenas relaciones con los vecinos, habla de un clima de paz y acercamiento, sin embargo, esto se ve subsumido en lo económico.

Lagos también habla de una integración en el ámbito económico, si bien su discurso no es explícito en torno al tema, la mayoría del texto tiene un sentido similar al de Frei Ruiz-Tagle, lo que se ve reflejado en el siguiente párrafo: "Eliminaremos los controles burocráticos que impidan la integración con los mercados financieros internacionales y la diversificación del mercado nacional, con la sola excepción, obviamente, de aquellas regulaciones prudenciales que protejan la integridad del sistema tributario y velen por la estabilidad y solidez del sistema financiero"32. ....y continúa, "Al mismo tiempo nos abriremos cada vez más a la realidad global, ampliando nuestra política hacia América, Asia Pacífico y Europa alcanzando con estas tres zonas acuerdos económicos estables y compartiendo con ellas la realización de ideales comunes"33.

Se puede ver en estos discursos el cambio de doxa en cuanto al vínculo internacional, la integración deja de ser entre países y pasa a ser una integración entre empresas vía Estados, como en la mayoría del mundo globalizado. El vínculo pierde el sentido social para encontrar un lazo en lo económico, es el campo económico el que nuevamente manifiesta su autonomización extendiéndose al campo social en el ámbito de las relaciones internacionales.

\section{Cambio de Doxa en el Campo Social}

\section{Del pueblo a la gente}

\footnotetext{
31 Frei Ruiz-Tagle.

32 Lagos Pagina 25.

33 Lagos Página 31.
}

Para Leda Berardi "El término gente comienza a implementarse en el gobierno de Patricio Aylwin con el lema "gana la gente", acuñado en la campaña electoral de 1989, en contraposición a pueblo, asociado a una cultura política previa. En una entrevista realizada a José Auth, sociólogo vinculado a la campaña electoral de EFRT, señala que "pueblo era una palabra guerrera, conflictiva, y la gente pedía consenso, paz". Al respecto, puntualiza que a partir de Aylwin "la gente es (considerada) "más gente" y "menos clase" en términos de su intervención en política, no en términos de sus condiciones de existencia. La apelación era más ciudadana que de clase, (es decir), gente alude al carácter de ciudadanía más que a condición social"34, Esto puede tener dos lecturas, por una parte la unificación en el concepto de las clases sociales en pugna y por otra la neutralización de un concepto que contribuía a la identificación de un grupo de la población y por tanto desde la perspectiva marxista contribuía a y la generación de una "clase para si", que participar como colectivo en política y de exigir sus derechos como era el pueblo en la doxa pre dictadura.

Frei Montalva como representante electo en el contexto dóxico predictatorial, parte su discurso dirigiéndose al "pueblo de Chile" y luego siempre se refiere a las personas que conforman la nación con este mismo vocablo. Salvador Allende por su parte alude al otro colectivo utilizando el concepto "pueblo" 46 veces, este es el concepto que mejor representa las características de aquello que estaba en el espacio de lo simbólico en la etapa pre dictadura, el pueblo no tenía vergüenza de autodenominarse pueblo y perderse en los que hoy se denomina la clase media baja. El concepto de pueblo tenía la fuerza simbólica de construir identidad colectiva en aquellos que estaban marginados del progreso del país. Hoy esos marginados se pierden en la nebulosa de la "gente pobre" como los denominaba Frei Ruiz-Tagle y Lagos en los gobiernos post dictadura.

Frei Ruiz Tagle en particular utiliza ambos conceptos indistintamente, pueblo y gente son

\footnotetext{
34 Berardi Drudi, Leda; "Legitimidad y Discurso Presidencial. Un análisis de los discursos de los Presidentes Eduardo Frei Montalva y Eduardo Frei Ruiz-Tagle.", en Revista Chilena de Semiótica, №1 1996.
} 
El cambio de doxa en Chile De la solidaridad al individualismo indiferente...

parte constitutiva de su discurso, sin embargo, más que considerar al pueblo como un actor en sí como lo hace Allende, por el contrario Frei siempre lo vincula a sus representantes poniendo a las personas representadas en un espacio secundario "La gente debe aprender a disciplinarse, a impulsar ciertos proyectos, a privilegiar ciertas acciones. Un pueblo informado es un pueblo lúcido que trabajará por sus propios objetivos. La gente intuye la actual oportunidad como una ocasión única. Cada vez que recorro el país, compruebo que la gente trabaja con optimismo y espera, con prudente sabiduría, que sepamos estar a la altura de las circunstancias". El otro uso que hace Frei del concepto pueblo se vincula a los pueblos originarios.

La utilización del concepto "pueblo" en el caso de Ricardo Lagos, si bien se repite 11 veces, 6 de ellas es generalmente empleado para hacer referencia a los pueblos originarios o indígenas y no al "pueblo de Chile". Utiliza de modo frecuente el concepto neutro de "gente" para hacer alusión a la población, así habla de "para toda la gente" cuando hace referencia a la masificación de los servicios públicos.

Cabe destacar que Lagos además ve a la "gente" como un recurso más de la producción planteando "Queremos una economía competitiva, estable y equitativa. Mantener las desigualdades es un escándalo moral y un enorme desperdicio del recurso más valioso de un país: su gente" 35

Ver al pueblo como un recurso o como un actor secundario bajo el concepto neutro de gente era impensable en la época previa a la dictadura, muy pocos hubiesen tolerado que se tratase de excluir a las personas de la construcción del país, todos y cada uno se sentían parte de esa construcción, hoy pocos se sienten parte de algún grupo en particular, los individuos son una masa sin identidad que mediante el voto delegan la responsabilidad en otros de la conducción del país.

\section{Políticas sociales}

Eduardo Frei Montalva plantea en su

35 Lagos Escobar Ricardo página 7. discurso que su programa de gobierno se propondría atender "principalmente a las personas de más bajos recursos" el eje de su conferencia en el plano de las políticas sociales está puesto en que "la característica esencial de las sociedades subdesarrolladas de Latino América y en Chile es la existencia de conglomerados humanos, cuya condición se ha definido con una palabra: marginalidad" 36 , el "atender" a este grupo marginado implica la incorporación no sólo en el plano económico sino que también el plano político, Frei Montalva continua diciendo "la realización del plan de desarrollo económico debe ir acompañado, simultáneamente de un proceso de desarrollo social, sin el cual aquel no sólo carecería de sentido sino que en las condiciones de la sociedad democrática moderna sería técnicamente imposible"37, esto también es la imagen en el espejo de la doxa pre dictadura en la cual no era posible concebir democracia sin la participación directa del pueblo.

Allende concibe la política social como una cuestión que no es posible separar de su programa integral de gobierno, por el contrario se planteaba como un elemento central de las transformaciones que su gobierno intentaba generar, en su discurso en vez de plantear un acápite denominado "política social"38 o "desarrollo social"39, como los otros discursos aquí analizados. El compañero presidente habla de "lograr las libertades sociales" y señala "Nuestro camino es instaurar las libertades sociales mediante el ejercicio de las libertades políticas, lo que requiere como base establecer la igualdad económica. Este es el camino que el pueblo se ha trazado, porque reconoce que la transformación revolucionaria de un sistema social exige secuencias intermedias" 40

Su propuesta revolucionaria consistía en un cambio de paradigma respecto a la política social , Allende señala "Vamos al socialismo por el rechazo voluntario, a través del voto popular, del sistema capitalista y dependiente cuyo saldo es una

\footnotetext{
36 Frei Montalva página 65.

37 Frei Montalva página 65.

38 Frei Montalva página 65.

39 Lagos Página XII.

40 Allende página 8.
} 
sociedad crudamente desigualitaria, estratificada en clases antagónicas, deformada por la injusticia social y degradada por el deterioro de las bases mismas de la solidaridad humana"41 aquí existe un reconocimiento explícito a que es el capitalismo el que genera sociedades desiguales y que la idea es cambiar ese modo de producción por otro que permita la justicia social.

Ambos presidentes predictadura conciben la política social como un elemento de incorporación no sólo en el plano social sino que también en el plano político, que el Estado diseñara estrategias de integración social implicaba también el diseño de estrategias de integración política. El pueblo conciente de sus derechos no aceptaba además que se marginara de los avances sociales, políticos y económicos del país ni esperaba que las clases altas acumularan lo suficiente para que "chorreara" algo para ellos.

En cambio, en la época post dictadura analizada, la política social está ligada al crecimiento económico, Frei Ruiz Tagle plantea que existe una "nueva oportunidad histórica" para el país, esta nueva oportunidad está vinculada con un crecimiento macroeconómico de un $6 \%$, aumento en las exportaciones, aumento de ahorro, bajas tasas de desempleo, aumento de las inversiones en el país y a una baja inflación, nótese que todos los aspectos señalados tienen una connotación económica, Frei Ruiz Tagle habla de Crecimiento con equidad y señala que es importante que cada chileno sepa "en que consiste esta nueva oportunidad. Es el modo de frenar a los oportunistas, a los del populismo prematuro, los nostálgicos de la polarización y la violencia. Todos los chilenos deben saber que la estabilidad política es una indispensable condición para el crecimiento económico. Todos, especialmente los más pobres, deben saber de qué manera el desarrollo social depende del crecimiento económico. La gente más humilde y sencilla debe distinguir a los que están al lado del progreso, de aquellos que tan solo provocan falsas querellas" ${ }^{42,}$ es interesante relevar el tono de advertencia, casi amenaza en el que Frei Ruiz Tagle se refiere a la estabilidad y a la negación de la demanda popular, la doxa post dictadura permite que un presidente se refiera al pueblo como "la gente más humilde y sencilla" y no como sujetos responsables con deberes y derechos. Lo increíble es que las personas a las que Frei Ruiz Tagle se refiere no hallan reaccionado, eso es explicado por la nueva doxa diría Bourdieu, una naturalización del la inmovilismo social y una aceptación dogmática a la idea de que no existe posibilidad de desarrollo social sin crecimiento económico. Se advierte además en el discurso una fuerte descalificación hacia cualquier tipo de organización que plantee algo distinto, cualquier intento por cambiar las formas a favor del pueblo es descalificada como populismo, la idea de que el pueblo vuelva a tener la relevancia histórica de antaño, es como si al sólo pensarlo se hiciere referencia a un demonio que hay que exorcizar del imaginario social chileno. Lagos Escobar por su parte también vincula crecimiento económico con desarrollo social y señala "el deseo de aprovechar el momento en que vivimos" haciendo alusión, al igual que Frei, a indicadores macroeconómicos. En relación a la pobreza señala "les invito a expandir al máximo nuestra capacidad económica, para que esa parte de la familia chilena que sufre de la pobreza se siente también a la gran mesa común, a compartir los frutos de la nación" ${ }^{43}$, de aquí se pueden extraer dos ideas, la primera y más importante es el reconocimiento implícito de las desigualdades sociales en el país y la segunda es que esas desigualdades se superan por medio del crecimiento económico en el marco del modo de producción capitalista. Sería impensable en el periodo predictadura que un militante del partido socialista hubiese realizado este discurso, la aceptación por parte de la población de estas contradicciones pudiesen explicarse por el cambio de doxa, a nivel de la personalidad de Lagos los cambios de más doctrinario a más pragmático, diría Mella, podrían ser explicados por la formación de preferencias adaptativas como una forma de disminuir la disonancia cognitiva entre preferencia individuales y la realidad; fenómeno al que Paul Veyne ha denominado "sobre adaptación a lo posible" 44

\footnotetext{
41 Allende página 4.

42 Frei Ruiz Tagle página 54
} 
El cambio de doxa en Chile De la solidaridad al individualismo indiferente...

\section{Educación}

En general la educación es siempre concebida como un elemento que permite el ascenso social, Bourdieu diría que en realidad es un elemento de reproducción dentro del espacio social.

Frei Montalva da cuenta de la relevancia de la educación como "instrumento de desarrollo cultural integral" ${ }^{\prime 45}$ aunque plantea que la política educacional de su gobierno "se orientará fundamentalmente a la formación de la persona como tal, restaurando plenamente los valores humanos para preparar una sólida base cultural común", agrega que ésta está en función de permitir "la entrada a la sociedad industrial" 46 esto es una diferencia respecto al gobierno que le sigue, donde Allende Gossi concibe la educación dentro de la antigua doxa, como un elemento esencial para el desarrollo del ser humano, que potencie sus habilidades innatas al servicio de otros, al servicio del pueblo, habla de "Un Chile en que todos los niños empiecen su vida en igualdad de condiciones, por la atención médica que reciben, por la educación que se les suministra, por lo que comen. Un Chile en que la capacidad creadora de cada hombre y de cada mujer encuentre cómo florecer, no en contra de los demás, sino en favor de una vida mejor para todos" 47 . Nótese el carácter solidario que denotan estas palabras, en esa época se estudiaba por todos y para todos, para hacer grande al país.

Después de la dictadura, los cambios dóxicos están asentados en la población y, a pesar de que Frei Ruiz-Tagle señala 8 veces a la educación en su discurso, la mayoría de ellas hace referencia a la educación como una forma de superar la pobreza, pero no por la necesidad de bienestar de las personas sino como un bien transable en los mercados, Frei dice: "La educación constituye para nosotros una de las tareas decisivas que debemos afrontar como nación. Mejorar su calidad es un imperativo moral, democrático y económico. Nótese el nuevo carácter imperativo económico que tiene la educación, más allá de lo moral y como componente de la democracia

\footnotetext{
45 Frei Montalva página 68.

46 Frei Montalva página 68.

47 Allende Página 4.
}

En la nueva doxa se incluye la educación dentro del Estado subsidiario y por tanto no responsable directo de la prestación del servicio educacional sino un ente que aporta recursos financieros (subvenciones) a otras instituciones para que lo hagan. La nueva doxa no ve en la educación el desarrollo del espíritu ni la necesidad de servir al país, la educación es, lo que es para los clásicos neoliberales, una herramienta de movilidad social.

El autodenominado presidente socialista post dictadura plantea que la educación es una de sus preocupaciones fundamentales y señala: "Como lo he señalado muchas veces, mi gobierno quiere poner su vista en el futuro. Tengo la convicción de que es en la educación donde se juega el futuro de Chile", sin embargo, este futuro esencial para el desarrollo del país se construye desde la subsidiariedad y la diferencia, ante lo cual sale al paso planteando: "Sueño con una educación igualmente buena en una escuela municipal muy modesta allá en el sur o en el mejor colegio privado del barrio alto de Santiago". De aquí podemos colegir que no le interesa eliminar las diferencias de dependencia sino las diferencias técnicas, pero como diría Bourdieu, lo importante aquí no es el tipo de enseñanza sino los lazos y los contactos que se establecen en el colegio. La nueva doxa acepta la propuesta de Frei y Lagos, no cuestiona lo que está por encima de lo técnico, es parte de la doxa aceptar las diferencias entre la educación segregada entre ricos y pobres, nadie se atrevería a plantear hoy algo como lo que se hizo durante la unidad popular de becar a los mejores alumnos de los colegios fiscales para que asistieran a colegios privados, eso hoy sería cuestionado o cuando menos considerado extraño.

\section{Trabajo}

Antes de la dictadura la organización sindical y la participación de los trabajadores en el mejoramiento de las condiciones económicas del país eran incuestionables, se trataba de crecer pero con justicia social. Ante esto Frei Montalva señalaba que "el objetivo fundamental de la política laboral consiste en incorporar a los trabajadores, en forma organizada, tanto en la formulación de 
los planes generales de gobierno como en su participación real en los beneficios de la actividad económica del país"48.

La opinión predictadura respecto al tema laboral se basa en un cuestionamiento a la relación Privados Estado, y se propone corregir lo que considera un error que no promueve un real desarrollo, Allende plantea que: "Las causas del atraso estuvieron -y están todavía- en el maridaje de las clases dominantes tradicionales con la subordinación externa y con la explotación clasista interna. Ellas lucraban con la asociación a intereses extranjeros, y con la apropiación de los excedentes producidos por los trabajadores, no dejando a éstos sino un mínimo indispensable para reponer su capacidad laboral"49. La doxa dominante apuntaba a la distribución de los ingresos y a un control de la ganancia de las clases dominantes.

La dictadura impuso profundas reformas laborales, iniciadas en 1978 y que fueron consolidadas en el nuevo Código Laboral dictado en 1987 y cuyo contenido hubiese sido imposible de presentar ante la población en el contexto dóico predictatorial, dado que esta habría reaccionado de tal manera que peligraría la estabilidad del país.

Frei Ruiz-Tagle, señala: "Una economía moderna requiere elevar la calidad de los empleos, perfeccionar sus instrumentos de capacitación y promover sistemas de relaciones laborales equitativos, adecuados a las necesidades de flexibilidad de los mercados de trabajo", aquí aparece por primera vez el concepto flexibilidad laboral, la norma afirmada por la violencia de todo tipo de la dictadura se asentó en la población y hoy en día la flexibilidad laboral es parte de lo que sucede en el plano de la producción, los trabajadores no ven alternativas, con la destrucción de los sindicatos y la ley del trabajo de José Piñera, la realidad laboral cambió, y hoy, no se cuestiona, como tantas otras cosas que hemos visto en este texto.

Lagos Escobar, hombre más político y menos empresario que su antecesor establece un consenso implícito con los postulados neoliberales en torno al tema laboral, así, él mismo plantea: "En la

\footnotetext{
48 Frei Montalva Página 71.

49 Allende Página 3.
}

pasada campaña electoral quedaron de manifiesto importantes convergencias en esta materia, lo que me hace ser optimista en cuanto a una rápida tramitación. Perfeccionar la normativa laboral dará un horizonte de estabilidad indispensable para tener también un mayor dinamismo económico". Esto da cuenta de la nueva doxa de consenso que prima en la época actual en donde un presidente socialista, encuentra puntos de convergencia con aquellos partidos que representan al empresariado chileno digno de análisis en otro trabajo. El cambio doxico queda en evidencia, la flexibilización laboral no es un tema a cuestionar por los socialistas que apoyan al gobierno. El pueblo no lee entre lineas como antes porque perdió los lentes, la nueva óptica aprecia el acuerdo entre Estado y empresarios, se cree en el "chorreo" puesto en el imaginario social por la dictadura.

\section{Violencia}

La Unidad Popular fue una época de muchas esperanzas, pero también de mucha violencia y temor, la constante amenaza de un golpe de Estado, la existencia de grupos armados de izquierda y de derecha hacían que el clima imperante en el país considerara la violencia como parte de los riesgos que debía correr la Unidad Popular. Allende estaba claro que la violencia política existía y se refería a ella de este modo: "Con todo, es mi obligación advertir que un peligro puede amenazar la nítida trayectoria de nuestra emancipación y podría alterar radicalmente el camino que nos señalan nuestra realidad y nuestra conciencia colectiva; este peligro es la violencia contra la decisión del pueblo". El compañero presidente advertía intuitivamente lo que vendría tres años después de su discurso y que le costaría la vida a tantos y a él mismo.

El Frei hace alusión a la violencia política como algo del pasado, como algo superado y que no puede volver, pero no porque la población se merece una mejor calidad de vida sino porque es una "indispensable condición para el crecimiento", así, este presidente utiliza la amenaza simbólica contra aquellos que pueden pensar que la violencia puede ser una alternativa para cambiar el sistema, Frei dice: "Cada chileno debe saber en qué consiste esta nueva oportunidad. Es el modo de frenar a 
El cambio de doxa en Chile De la solidaridad al individualismo indiferente...

los oportunistas, a los del populismo prematuro, a los nostálgicos de la polarización y de la violencia. Todos los chilenos deben saber que la estabilidad política es una indispensable condición para el crecimiento económico". Sus palabras hablan por si solas.

Es el delito, no la violencia política lo relevante, en el periodo, pero la instalación de este concepto no es neutra, es en la época de Frei donde se comienza a relevar el delito como un elemento central de la opinión pública, pero este concepto está instalado por los medios de comunicación como un substituto del terrorista de la dictadura, el delincuente permite justificar políticas represivas y de control social. La nueva doxa incorpora al delincuente como el nuevo enemigo del cual defenderse y es funcional a la idea de individualismo, la desconfianza respecto del otro se instala durante la dictadura, algunos temían ser denunciados, otros temían a los terroristas. Eso permitió la atomización, hoy es el delito el gran tema que genera esa atomización, no es bueno conversar con alguien en la calle, no es bueno confiar en otros desconocidos y por sobre todo los pobres son todos potenciales delincuentes.

El presidente socialista Ricardo Lagos tiene la misma postura que su predecesor, y refiere a la violencia como algo del pasado diciendo: "La nueva época que vamos a inaugurar se basa en el principio de la cooperación y la solidaridad. Hay que renunciar al uso de la amenaza o la violencia, incluida la violencia verbal, que tanto perjudicó los procesos de transformación que se intentaron en el pasado". Este discurso es representativo de la nueva doxa, se excluye la violencia como una forma legítima de cambiar el curso de la historia, esto más allá de que se esté de acuerdo o no con ello, no era así en la doxa anterior. Por otra parte también rescata el delito como un elemento preocupante durante su mandato y utilizando un tono de amenaza plantea que "Dije en mi campaña presidencial que mi gobierno sería firme en el castigo a los delincuentes"

\section{Conclusiones}

La "actitud dóxica" dice Bourdieu "no supone felicidad, supone sumisión corporal, sumisión inconsciente, lo cual podría poner de manifiesto mucha tensión interiorizada, mucho sufrimiento corporal" 50 . Las personas que habitan Chile hoy sufren esa sumisión inconciente, no son felices pero no saben porque, se niegan siquiera a preguntarse que es lo que le sucedió a nuestra sociedad después de la dictadura. Algunos pensaron con que bastaba con una comisión que en 1990 evacuara el informe Rettig para avanzar en la reconciliación del país, otros señalaban que sumado a este debía haber justicia, no sólo verdad, cuestión que hasta hoy ha tenido pocos avances, pero muy pocos se dan cuenta que la dictadura se instaló en el imaginario social, el Chile colectivo lleva la dictadura en sus prácticas cotidianas, se configura en sus relaciones mediado por ella. Quizás sólo los más jóvenes han logrado darse cuenta e intentar modificar de algún modo las cosas, pero el mundo adulto intenta encasillarlos para que reproduzcan las prácticas dictatoriales, reproduciendo a su vez dichas prácticas.

La relevancia del texto está en mostrar por medio del análisis del lenguaje utilizado en los discursos presidenciales la evolución doxica del país. Bourdieu al referirse a la relevancia del lenguaje señala que "La capacidad de hablar por estar inscrita en el patrimonio biológico, es universal y por lo tanto no distintiva" 51 , pero habla también de la necesidad de una sociología estructural de la lengua que permita analizar la relación entre sistemas diferenciados lingüísticamente relevante desde una perspectiva sociológica y sistemas estructurados de diferencias sociales. El lenguaje que refleja la doxa anterior a la dictadura: Incorpora elementos tales como, público, expropiar, cooperativa, popular, pueblo y bienestar de la nación. La mayoría de esos conceptos hacen alusión a lo colectivo, a lo solidario a la construcción de país en conjunto. La nueva doxa en cambio, incorpora los antónimos de los conceptos anteriores, lo privado, privatizar, subsidio individual, individuo, gente e intereses de mercado. Y, como diría Bourdieu "Se puede temer que las estructuras de este sistema se transformen en inestabilidad crónica, inscribiéndose en los cerebros bajo la forma de estructuras cognitivas, contribuyan a

\footnotetext{
50 Bourdieu, Pierre \& Eagleton, Terri "Doxa y vida ordinaria”, New Left review, enero del 2000, página 231.

51 Bourdieu, Pierre; "Qué significa hablar", ediciones Akal, Madrid 1985. Páginas 28 y 29.
} 
producir un hombre nuevo (como se decía en otro contexto) calculador universal llevado al cinismo oportunista en política y en otra parte, y a la inconstancia y también al individualismo por no decir egoísmo, favorecido por la corrupción de la persona y la destrucción de las solidaridades"52. Las elites nacionales, económicas, políticas y algunos intelectuales tienen la idea de que la política de consensos es lo que efectivamente ha mantenido la estabilidad en la sociedad chilena, pero esto no es cierto, es la doxa modificada por la dictadura lo que hasta hoy mantiene una estabilidad aparente.

\section{Referências}

Allende Grossi, Salvador. La Vía Chilena al Socialismo. Primer Mensaje al Congreso Pleno, 1970

Aylwin, Mariana et. Al. Chile en el siglo XX. Santiago, Editorial Planeta, 1990.

Berardi Drudi, Leda. Legitimidad y discurso presidencial: un análisis de los discursos de los Presidentes Eduardo Frei Montalva y Eduardo Frei Ruiz-Tagle. Revista Chilena de Semiótica, n.1, 1996.

Bourdieu. P.; T. Eagleton. Doxa y vida cotidiana: una entrevista. In: Zizek, S. (comp.), Ideología: un mapa de la cuestión. Buenos Aires: FCE, 2003.

Bourdieu, Pierre. Los usos sociales de las ciencias. Buenos Aires, Argentina, 2000.

Bourdieu, Pierre. Razones prácticas, sobre la teoría de la acción. Barcelona, Editorial Anagrama, 2002.

Bourdieu, Pierre. La distinción, criterios y bases sociales del gusto. Madrid: Editorial Taurus, 1998.

Bourdieu, Pierre; Eagleton, Terri. Doxa y vida ordinaria. New Left Review, enero del 2000.

Bourdieu, Pierre. El sociólogo y las transformaciones recientes de la economía en la sociedad. Buenos Aires: Editorial Libros de Rojas, Universidad de Buenos Aires, 2000.

Bourdieu, Pierre. Cosas dichas. Buenos Aires, Editorial Gedisa, 1987.

Bourdieu, Pierre. Intelectuales, política y poder. Buenos Aires, EUDEBA, abril 2000.

Bourdieu, Pierre. Los usos sociales de sociales de la

52 Bourdieu, Pierre; "El sociólogo y las transformaciones recientes de la economía en la sociedad", Libros de Rojas, Universidad de Buenos Aires. 2000 ciencia. Buenos Aires, Editorial Nueva Visión, 2000.

Bourdieu, Pierre. Qué significa hablar. Madrid: Ediciones Akal, 1985.

Bourdieu, Pierre. La dominación masculina. Barcelona, Editorial Anagrama, 2000.

Cavallo, Ascanio; Salazar, Manuel; Sepúlveda, Oscar. La historia oculta del régimen militar, memoria de una época 1973-1988. Santiago, Editorial Grijalbo, 1997

Diccionario de filosofía. Disponível em: http://www. filosofia.com.mx/index.php?/perse/archivos/la_jerga_ del_filosofo4/

Diario La Nación, 26 de Septiembre de 2004"

Eyzaguirre, Jaime. Breve historia de las frontera de Chile. Santiago, Editorial Universitaria, 1979.

Frei Montalva, Eduardo. Discurso presidencial. 21 de mayo de 1965

Frei Ruiz Tagle, Eduardo. Mensaje de presidencial. 21 de Mayo de 1994. Gobierno de Chile. 1994

Frei Ruiz Tagle, Eduardo. Plan de trabajo 1996. Chile, Viña del Mar, 1996.

Garretón, Manuel Antonio; Moulian, Tomás. La Unidad Popular y el conflicto político en Chile". Santiago, Ediciones Minga, 1983.

Lagos Escobar, Ricardo, Discurso presidencial. 21 de mayo de 2000.

Lechner Norbert. Los nuevos perfiles de la política. Revista Nueva Sociedad, p.180-181, 1994

Massardo, Jaime; Suarez-Rojas, Alberto. América Latina mosaico cultural. Paris, Editorial Elipses, 2005.

Mella, Marcelo. Carácter y transformaciones del liderazgo de Lagos. Disponivel em: http://www.iigov. org/wp/attachment.drt?art=688758 Acesso em: mar 2005.

Rowart, Malcom. The emerging role of the state in Latin America. Public Management, v.1, n.2, p.261-287, 1999.

Solis, Luis; Rojas, Francisco. La integración Latinoamericana. Observatorio para las relaciones Europa América Latina, FLACSO, Secretaría General, Editorial Juricentro, San José, Costa Rica. Página 138

Vargas Aguirre, Mónica; Políticas sociales y trabajo social un análisis histórico desafíos, dilemas y propuestas, Ponencia presentada al Congreso Internacional "Políticas Sociales para un Nuevo Siglo", Universidad del Bío, 21 al 24 de Noviembre, Concepción Chile. Disponivel em: http://www.ubiobio.cl/cps/ponen$\mathrm{cia} / \mathrm{doc} / \mathrm{p} 14.5 . \mathrm{htm}$ 